\title{
Die Methoden zur Aufschließung der weichen Geburtswege und ihre Indikationen.')
}

\section{Von H. Fehling.}

M. H.! In den letzten zwei Jahrzehnten hat die Zahl der zur Erweiterung der Cervix verfügbaren Methoden so stark zugenommen, daß man nicht selten erlebt, daß infolge dieses Uebersegens zum falschen Mittel gegriffen wird, da naturgemäß mit der Zahl der Mittel auch die Indikationsstellung für das einzelne Mittel schwieriger geworden ist. Ich halte es daher nicht für unwichtig, hier im Kreise der Praktiker diese Frage zur Besprechung zu stellen, in der Absicht, auch die von Ihnen in der Praxis gemachten Erfahrungen in der Diskussion zu vernehmen.

Unter den Indikationen stehen natürlich weit voran Blutungen. Blutungen in jeder Zeit der Schwangerschaft werden dann die Indikation zum Eingriff geben, wenn trotz genügend langer Ruhe, Anwendung von Narcotica etc. die Blutung nicht steht, oder wenn sie eine lebensgefährliche Höhe erreicht. Auch hier spielt soziale Indikation etwas herein. Ich werde naturgemä $\beta$ bei einer Arbeiterin, die zum Hauswesen mit verdienen muß, zumal wenn schon Kindersegen in genügender Fülle vorhanden, mich leichter zum Eingriff entschließen als da, wo die Frau sich wochen- und monatelang schonen kann und mit Bangen der Erhaltung ihres ersten Kindes entgegensieht.

Ich will gleich hier anführen, daß ich es für falsch halte, bei abzestorbsnem Ei einzugreifen, solange nicht Blutung, Jauchung, Fieber dazu drängt. Ein abgestorbenes Ei kommt schließlich immer von selbst, infiziert die Trägerin nicht, wenn nicht von außen Keime importiert werden. Wozu also hier eingreifen?

Ganz anders steht es bei zersetztem Uterusinhalt, wenn zur Blutung in der Schwangerschaft sich Jauchung und Fieber gesellt. Meist wird man hier mit Recht annehmen, daß unreine Hände, und zwar meist verbrecherischerweise, tätig waren. Aber auch gut gemeinte Manipulationen, wie Tamponade, können bei Anwesenheit bestimmter Keime im Genitalkanal Zersetzung und Fieber hervorrufen. Nur recht selten wird man der Annahme Raum gebən dürfen, daß ohne irgendwelches Zutun, selbst ohne die Finger der Schwangeren, Keime von der Scheide und Cervix eingewandert sind und schwere Zersetzung bedingt haben. Zersetzung des Uterusinhalts, gekennzeichnet durch Jauchung und Fieber, bedingt also unbədingt möglichst rasche Entleerung des Uterus.

Viel schwieriger sind die Indikationen festzulegen, die durch vorhandene oder drohende Lebensgefahr der Mutter gegeben werden, also Tu berkuloseder Lungen, Herzfehler, sowie Nephritis etc. Ich will diese heikle Frage hier nicht aufrollen, sie würde mich zu weit führen; ich betone nur allgemein,

2) Vortrag in der Gynäkologischen Sekttion der Versammlung der Naturforscher und Aerzte in Karlsruhe. 
daß hier von Fall zu Fall die Indikation gestellt werden muß; und zwar soll jeder, auch der Kliniker, einen erfahrenen Internen zuziehen.

Leichter sind wieder die Fälle zu entscheiden, wo gegen Ende der ersten oder in der zweiten Hälfte der Schwangerschaft akutes Hydramnion die Mutter bedroht, wo bei Nephritis ein Oedem der Vulva zu Gangrän führen kann, oder wo bei bedrohlichen Beschwerden durch ein vorgeschrittenes Herz- oder Lungenleiden man bei lebensfähigem Kind durch einen frühzeitigen Eingriff der Leidenden ein paar Wochen früher Erleichterung verschafft.

Was die Methoden der Erweiterung der weichen Geburtswege anlangt, so kommen für uns sieben in Betracht; ich bespreche sie gesondert, je nachdem sie in der ersten oder zweiten Hälfte der Schwangerschaft zur Verwendung kommen.

Für die erste Hälfte der Schwangerschaft kommen in Betracht: Laminaria, Hegarsche Stifte, Diszision, Bossi.

Laminaria ist wohl unser ältestes Mittel, und ich gehöre zu denen, die es heute noch verwenden und keine schlechten Erfahrungen damit gemacht haben; der Laminariastift hat die besondere Wirkung, die Cervix zu erweitern, sie ödematös zu erweichen und Wehen anzuregen. Die Indikation geben alle die Fälle, wo man sich entschließt, in den ersten Monaten den Abort einzuleiten, ohne daß schon Zeichen der Erweiterung da sind; dann schwere Blutungen, wo das Ostium interuum noch nicht offen ist.

Kontraindikation: Uebelriechender oder infektiöse Keime enthaltender Abgang, Fieber. Ratsam ist heutzutage auch für Sie, meine Herren, in allen Fällen vor dem Eingriffe das Sekret der Vagina und Cervix zu entnehmen und bakteriologisch untersuchen zu lassen.

Ausführung: Der Laninariastift, dickste vorhandene Nummer, wird fünf Minuten in 5\% igem Karbolwasser gekocht; während dieser Zeit Desinfektion der Vulva, Vagina und Cervix in üblicher Weise; Erweiterung der Cervix bis Hegar 9 oder 10, Biegen des weichen Stifts (mit sterilen Handschuhen), der Uterusaxe entsprechend, Einführen mit kräftiger Kornzange. Das Herausgleiten läßt sich leicht verhindern durch Tamponade der Cervix mit schmaler Gaze, dann der Scheide mit breiter Gaze. Der Stift bleibt 24 Stunden liegen; Temperaturmessung; dann Herausnehmen in Narkose. Macht die Herausnahme Schwierigkeit, dann Diszision der strikturierten, meist dem Ostium internum entsprechenden Stelle neben dem Stift. Nach Herausnehmen Desinfektion der Uterushöhle. Dringt der Finger nicht genügend leicht ein, dann weitere seichte Diszision der hemmenden Stelle mit dem gebogenen Metrotom. Ich bin auf diese Weise stets mit einem Stift ausgekommen.

An die Ausräumung des Eies schließt sich eventuell die Ausschabung an.

Die Erweiterung mit Hegarschen Stiften genügt für diese Fälle nicht, wo man Fingerdurchgängigkeit erzielen will. Mehr als bis Hegar 12 zu gehen, halte ich für gefährlich, weil dann leicht das Cervixrohr platzt und infizierbare parametrane Verletzungen entstehen. Die Indikation für Hegarsche Stifte ist gegeben, wenn das $\mathrm{Ei}$ nachweislich fort ist und nur Reste davon zurückgeblieben sind, also nur eine Ausschabung nötig ist. Die Gefahr der Perforation mit Hegarschen Stiften, welche von anderer Seite hervorgehoben wurde, halte ich für fast ausgeschlossen, wenn der den Spiegel haltende assistierende Gehilfe (oder die Gehilfin) mit der anderen Hand den Uterus kontrolliert. Viel gefährlicher quoad perforationem ist Sonde und Kornzange. Für die Ausschabung genügt meist Erweiterung bis 9 oder $10 \mathrm{~mm}$. Hat man schon früher Schwierigkeit bei Durchführung der Dilatatoren, dann schneidet man die unnachgiebige Stelle besser mit dem Metrotom ein.

Die bilaterale Diszision der Cervix als Verfahren allein (um die Cervix genügend zu erweitern) ist heutzutage nicht mehr empfehlenswert. Entweder erzielt man keine genügende Erweiterung für Fingerdurchgängigkeit, oder die Blutung wird durch Verletzung eines Uterinalastes gefährlich stark. Die Diszision als solche hat demnach keine Indikation mehr. Will man nur schneidend vorgehen, dann tritt die Hysterotomia anterior an ihre Stelle.
Endlich gehört die Erweiterung der Cervix nach Bossi für bestimmte Fälle hierher.

Indikation: Zersetzter Uterusinhalt und Fieber und damit die Aufgabe, den Uterus rasch und gefahrlos in einer Sitzung zu entleeren. Hier sind Laminaria, Hegarsche Stifte, ebenso Gazetamponade kontraindiziert. Wir haben in Klinik und Poliklinik bəi einer großen Reihe von Fällen gerade bei putridem Abort Bossi verwandt und sind äußerst zufrieden mit den dadurch erzielten Erfolgen, während uns bisher kein gleichwertiges Mittel zur Verfügung stand. Gerade auch für den in der Praxis alleinstehenden Arzt scheint mir das Instrument ganz besonders empfehlenswert zu sein. Allerdings muß man ein gutes Instrument verwenden, wie das letzte Modell von Bossi, das ich Ihnen hier vorzeige und das alle die angegebənen Modifikationen weit übertrifft.

Ausführung: Narkose, übliche Desinfektion von Scheide und Cervix, Anhaken der mit Rinnenspekulum freigelegten Portio, Einführen der Spitzen ohne Kappen; langsame Erweiterung (fünf bis zehn Minuten) unter stetem Zug an der Portio, bis auf $3 \mathrm{~cm}$, wobei meist der Finger durchdringen kann. Dann Ausräumung der Uterushöhle manuell, mit nachfolgender Ausschabung, heiße desinfizierende Uterusspülung und meist nachfolgender Ausstopfung des Uterus mit Vioformgaze, die mit $10 \%$ igem Kampferöl imbibiert ist.

Schwere Verletzungen, wie sie bei Anwendung im neunten oder zehnten Monat vorkommen, haben wir hierbei nie gesehen.

Ich sehe das Instrument als eine wertvolle Bereicherung des Instrumentariums an, zur raschen und schonenden Vollendung septischer Aborte, die ja überall, besonders aber in den Großstädten, so häufig dem Arzt Schwierigkeit machen.

Für die zweite Hälfte der Schwangerschaft kommen in Betracht Gazetamponade, Bossi, Metreuryse, Hysterotomia anterior, bilaterale Cervixinzisionen.

Die Gazetamponade hat ein verhältnismäßig kleines Anwendungsgebiet. Sie ist ein relativ schwaches Mittel, Erweiterung und damit Wehen hervorzurufen, und soll daher nur angewandt werden, wo nicht rasche Erweiterung der Cervix not tut. Sie paßt besonders bei lange dauernden Blutungen, wie sie durch endometritische Vorgänge bedingt werden, welche die Frau erschöpfen und wo die Cervix sich sehr allmählich erweitert.

Während ursprünglich Hof meier Jodoformgaze empfahl, wende ich nur noch Vioformgaze an: Im Rinnenspekulum wird nach vorgängiger Desinfektion der Vulva und Scheide die Portio angehakt, die Cervix mit Playfair ausgerieben und nun so viel $4-6 \mathrm{~cm}$ breite Gaze mit einem passenden Gazestopfen über das Ostium internum heraufgebracht, wie hineingeht. Es ist peinlich darauf zu achten, daß die Gaze nicht an Schamlippen oder Scheide anstreift, zur Sicherheit zieht man Gummihandschuhe an. Die Gaze soll mindestens 24, aber nicht über 36 Stunden liegen bleiben, dabei genaue Temperaturbeobachtung. Dann nimmt man sie heraus und überläßt entweder die weitere Ausstoßung der Natur, oder man wendet ein weiteres Verfahren wie Metreuryse an. Recht selten wird eine Wiederholung der Gazeeinführung nötig sein.

Die Metreuryse ist das Hauptmittel zur Einleitung der Geburt in der zweiten Hälfte der Gravidität. Der Vorteil der Metreuryse besteht in dem Wegbleiben von Verletzungen beim Einführen, in der sicheren Desinfektionsmöglichkeit des Apparates, der Möglichkeit, den Metreurynter lange liegen zu lassen, und der sicheren Wirksamkeit.

Die Indikationen zur Anwendung sind für mich gegeben in erster Linie zur Einleitung der künstlichen Frühgeburt bei engem Becken, welche ich auch jetzt noch für den Praktiker reserviere; möglich, daß das Pituitrin, sobald wir größere Erfahrung darüber haben, diesen Wirkungskreis etwas einschränken wird. Dann kommen all die anderen Indikationen der Einleitung der Frühgeburt bei Krankheiten der Mutter, des Eies, Placenta praevia, zur weiteren Anregung der Geburt nach Gazetamponade etc.

Zur Verwendung kommt ein Sortiment von vier Metreuryntern von Geigenform, welche ich seinerzeit nach den Barnesschen modifizierte. Es kommt stets das größtmögliche Exemplar zur Verwendung, nachdem zuvor festgestellt, wieviel 
Wasser sich einspritzen läßt. Die Einführung geschieht mit densılben Kautelen wie bei Gazeeinführung. Es wird aber nicht sofort die möglichst starke Füllung angewandt, sondern der Ballon erst nach sechs bis zwölf Stunden ad maximum gefüllt. Der Metreurynter kann $24 \quad 48$ Stunden liegen bleiben. Nach mindestens zwölfstündigem Liegen kann Dauerzug damit verbunden werden; nicht eher, damit nicht der Metreurynter ausgestoßen wird, ehe er genügend Wehen hervorgerufen hat. Der Metreurynter dient nicht nur zur Erweiterung der Cervix, sondern regt auch Wehentätigkeit an, ähnlich der Laminaria. Gənaue Temperaturbeobachtung. Ist nach seiner Herausnahme oder Ausstoßung das Ostium internum noch nicht genügend erweitert oder nicht genügend Wehentätigkeit erwacht, so wird die nächst größere Nummer eingeführt. Am besten ist, wenn dadurch die Wehentätigkeit so in Gang gebracht wird, daß kein weiteres Verfahren als etwa später Blasensprengung nötig wird. Ich möchte sagen, was der Laminariastift in den ersten drei Monaten, das leistet in der zweiten Hälfte der Gravidität der Metreurynter. Dem Arzt ist damit ein absolut sicheres Mittel in die Hand gegeben schonend und aseptisch die Geburt in Gang zu bringen.

Auch an dieser Stelle muß ich nochmals des Bossischen Erweiterungsinstrumentes gedenken. Ich stehe nicht auf dem Standpunkt, wie er früher von der Leopoldschen Schule vertreten wurde, das Instrument in ausgedehntem Maße zur Erweiterung der Cervix anzuwenden, um den Fortgang der Geburt überhaupt zu fördern.

Auch in der zweiten Hälfte der Gravidität ist das Anwendungsgebiet des Bossi für mich eng begrenzt. Ich empfehle nur da die Anwendung, wo Gazetamponade und Metreurynter im Stich gelassen haben und wo eine dringende Indikation vorliegt, rasch die Geburt fertig zu machen, also bei Blutungen, Fiebər.

Eine weitere Indikation ist für den Praktiker die Eklampsie. Ich habe die Ueberzeugung, daß die schleunige Entbindung in der Klinik im ganzen die Prognose für Mutter und Kind etwas gebessert hat. So treten auch an den Arzt draußen Fälle heran, wo die Schwere des Falles, die Häufigkeit der Anfälle es wünschenswert machen, die Geburt zu vollenden. Ist hier noch Zervikalkanal vorhanden und paßt der Fall nicht für die von Dührssen vorgeschlagene Ballontamponade mit zervikalem Kaiserschnitt, dann ist langsame, auf eine halbe bis eine Stunde ausgedehnte Erweiterung der Cervix mit Bossi angezeigt. Wenn dadurch eine genügende Erweiterung des Ostium internum erzielt wird, kann die Geburt mit Zange oder Wendung vollendet werden. Die Ausführung geschieht unter denselben aseptischen Kautelen, wie oben beschrieben. Ist schon Fingerdurchgängigkeit vorhanden, so werden die Spitzen des Instrumentes mit der Kappe bedeckt eingeführt.

Bei vorsichtiger Anwendung wird der Arzt so weniger schwere Zerreißungen erleben, als wenn er bei enger Cervix das Eindringen gewaltsam mit der Hand erzwingt.

Eine wesentliche Bereicherung der Methoden zur Aufschließung der Geburtswege hat uns Dührssen durch den von $\mathrm{ihm}$ so genannten vaginalen Kaiserschnitt gegeben; besser als Hysterotomia cervicalis anterior zu bezeichnen. Die Ueberlegung zeigte, daß die infravaginalen Inzisionen der Cervix nur ein begrenztes Anwendungsgebiet habon; daß aber die tiefen Cervixinzisionen, wie sie früher Dührssen angab, ihre großen Gefahren haben. Anderseits lehrten uns die gynäkologischen Operationen, wie leicht man nach Abschieben der Blase durch mediane Inzision der Cervix sich die Uterushöhle zugängig machen kann; so kam Dührssen sohr logisch auf den Vorschlag, dieses Verfahren auch in der zweiten Hälfte der Schwangerschaft zur raschen Entleerung der Gebärmutter zu benutzen. Die Methode verlangt immerhin gynäkologische Uebung, gute Assistenz, gute Beleuchtung etc., sodaß ich nicht rate, daß der Praktiker das Verfahren allgemein adoptiert; wenn ich auch gern anerkenne, daß es schon mit Erfolg in der Praxis ausgeführt wurde. Die Hauptindikation ist die Eklampsie, welche ja kaum je vor der zweiten Hälfte der Schwangerschaft auftritt; dann plötzliche Erstickungsgefahr der Mutter bei Herz- und Lungenleiden, zuweilen auch Nephritis, wo ein langsames und längere Narkose verlangendes
Verfahren nicht angezeigt ist. Von manchen Autoren wurde auch Lebensgefahr des Kindes durch Nabelschnurvorfall bei engem Muttermund als Indikation angegeben. Dagegen kann ich die Entleerung des Uterus durch Hysterotomia anterior in der ersten Hälfte der Gravidität als berechtigtes Verfahren nicht anerkennen, ebenso ist für mich auch Placenta praevia keine Indikation; sie ist auch von den meisten wieder verlassen worden. Auch schwere Infektion des Uterus kann die Indikation geben, wenn die anderen Verfahren voraussichtlich zu lange Zeit in Anspruch nehmen würden. Kontraindiziert ist die Operation bei räumlichem Mißverhältnis des Beckens; in diesem Falle greift man besser zum Kaiserschnitt. Zur Operation, Narkose eingeschlossen, braucht man mindestens drei, eventuell vier Assistenten, was schon die Ausführung in der Privatpraxis erschwert. Blutig ist meist nur der Querschnitt in der Scheide, um die Portio vom Scheidengewölbe zu trennen, das Hinaufschieben der Blase geht meist leicht, ohne größere Blutung, ebenso die Inzision in der vorderen Cervixwand, welche bei großen Früchten eventuell über das Ostium internum fortgesetzt werden muß. In einzelnen Fällen habe ich, wenn noch nicht genügend Raum war, auch noch eine mediane Inzision der hinteren Lippe bis zum Vaginalansatz hinzugefügt. Darauf folgt die Entwicklung der Frucht, meist durch Wendung, seltener durch Zange; nun folgt die Naht der Cervixwand und darauf die der Scheide.

Mit der Expression der Plazenta wartet man womöglich bis zum Schlusse der Operation. Die Aufnahme der Operation in die Reihe der geburtshilflichen Operationen bedeutet entschieden einen Fortschritt, anderseits gab sie auch Veranlassung zur besseren Ausbildung der übrigen Methoden.

Nur anhangsweise möchte ich hier noch der nultiplen Inzisionen der infravaginalen Cervix gedenken, welche, am richtigen Platz angewandt, segensreich wirken. Ihr Indikationsgebiet sind die Fälle von rigidem, derbem, undehnbarem Muttermund, besonders nach alter Genorrhoe, langer Aetzbehandlung, Portioamputation, entstanden, zuweilen auch grundlos, so z. B. bei alten Erstgebärenden. Ist hier in der Eröffnungszeit eine gute Wehentätigkeit vorhanden, Schlag auf Schlag folgende sehr schmerzhafte Wehen, die Blase gesprungen, und sieht man zumal in der Wehe sich den Muttermund derb spannen, die Oeffnung manchmal enger werden (Krampfwehen der Alten), dann paßt die Inzision. Die Natur legt uns eigentlich schon die häufigere Anwendung nahe, bei jeder Geburt entstehen seitliche Cervixrisse, und doch inzidiert man häufiger Hymen oder Damm als die Cervix. Die Inzision wird mit einem gebogenen, geknöpften Metrotom vorgenommen, besser und sicherer mit einer langen Cooperschen Schere. Die darauf folgende Blutung ist selten stark und gibt kaum je zur Tamponade Anlaß.

Fasse ich kurz zusammen, so empfehle ich für die erste Hälfte der Gravidität den Laminariastift, bei Zeitmangel Bossi; in der zweiten Hälfte entsprechend Metreuryse und Hysterotomia anterior.

Ich würde mich freuen, meine Herren, wenn mein Vortrag Thnen Veranlassung geben würde, Ihre besonders in der Praxis damit gemachten Erfahrungen auszutauschen. 